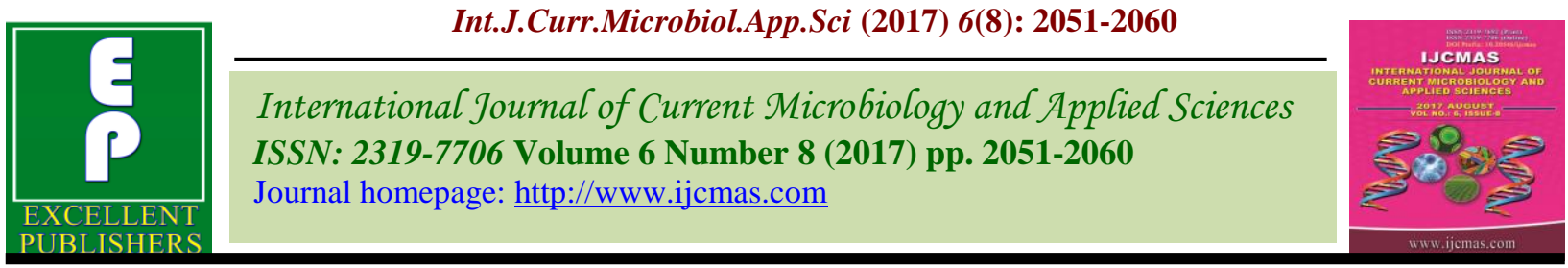

Original Research Article

https://doi.org/10.20546/ijcmas.2017.608.244

\title{
Utility of AhTE Markers for Genetic and Genomic Studies in Groundnut (Arachis hypogaea L.)
}

\author{
A.A. Hake and R.S. Bhat* \\ Department of Biotechnology, University of Agricultural Sciences, Dharwad, India-580 005 \\ *Corresponding author
}

A B S T R A C T

\begin{tabular}{|c|c|}
\hline Keywords & $\begin{array}{l}\text { Recombinant inbred line (RIL) and mutant populations of groundnut were employed to } \\
\text { assess the utility of Arachis hypogaea transposable element (AhTE) markers, which detect }\end{array}$ \\
\hline $\begin{array}{l}\text { AhTE Markers, } \\
\text { Arachis } \\
\text { hypogaea, } \\
\text { AhMITE1. }\end{array}$ & $\begin{array}{l}\text { the transposition polymorphism of Arachis hypogaea miniature inverted-repeat } \\
\text { transposable element (AhMITE1), for the genetic and genomic studies to enhance the } \\
\text { groundnut productivity. Of the two alleles (with and without AhMITE1 insertion) at each } \\
\text { locus, the latter was more frequent }(0.56) \text { across } 79 \text { AhTE markers among the genotypes. }\end{array}$ \\
\hline Article Info & AhTE markers. Similarly, the AhTE markers from the A genome showed higher $H_{O}$ when \\
\hline $\begin{array}{l}\text { Accepted: } \\
\text { 19 June } 2017 \\
\text { Available Online: } \\
10 \text { August } 2017\end{array}$ & $\begin{array}{l}\text { compared to those from B genome. Mutant population in general showed the higher } \\
\text { heterozygosity over the RIL population. AhTE markers displayed as high as } 65.82 \% \\
\text { polymorphism, based on which the genotypes could be classified into two groups. Thus, } \\
\text { the study indicated the usefulness of the AhTE markers in the genetic and genomic studies } \\
\text { in groundnut. }\end{array}$ \\
\hline
\end{tabular}

\section{Introduction}

Groundnut (Arachis hypogaea L.) has a genome of $2.8 \mathrm{~Gb}$, and the diploid progenitors have been sequenced (Bertioli et al., 2016; Chen et al., 2016) for hastening the application of genomics in groundnut breeding for various traits to enhance the productivity (Bevan et al., 2017). Currently, genomics is providing new foundations and genomic innovations for crop-breeding systems in many crops (Bevan et al., 2017; Yuan et al., 2017). Of them, the development and use of DNA markers is the frontrunner with significant applications in developing new crop varieties with considerable genetic gain (Yuan et al., 2017). Though several types of marker systems are available in groundnut, most of them suffer from low polymorphism especially in cultivated groundnut (Song et al., 2010; Khera et al., 2013) over the wild types (Moretzsohn et al., 2009), which limits their application in crop improvement (for review, Holbrook et al., 2011).

Arachis hypogaea transposable element (AhTE) markers, which detect the transposition polymorphism of Arachis hypogaea miniature inverted-repeat transposable element (AhMITE1), have been developed in groundnut (Bhat et al., 2008; Gowda et al., 2010; Shirasawa et al., 2012a). Due to the large number and variation for transpositional activity of AhMITE1, AhTE markers show considerably high (27.4\%) 
polymorphism (Shirasawa et al., 2012a; Kolekar et al., 2016). In this study, an effort was made to analyze the population genetic parametersusing AhTE markers among a mutant population and a recombinant inbred line (RIL) population of groundnut to assess the utility of AhTE markers in genetic and genomic studies.

\section{Materials and Methods}

Two types of populations; the first consisting of the RILs (432) of TMV $2 \times$ TMV 2-NLM, and the second consisting of mutants and their parents were used for assessing the genetic features of the AhTE markers. The AhTE markers used in this study were developed by (Shirasawa et al. 2012a and 2012b). Total genomic DNA was extracted from young leaves using modified cetyltrimethyl ammonium bromide (CTAB) method (Mace et al., 2003). A total of 79 AhTE markers were used for genotyping. PCR reactions were carried out with 50 ng of template DNA, 5 pmol of each primer, 10X of Taq polymerase buffer [500 mMKCl, $100 \mathrm{mM}$ Tris-HCI ( $\mathrm{pH}$ 8.5], $2.0 \mathrm{mM}$ of $\mathrm{MgCl}_{2}, 0.25$ $\mathrm{mM}$ of dNTPs and $0.15 \mathrm{U}$ of Taq polymerase in a total volume of $10.0 \mu \mathrm{l}$. PCR was performed in Veriti 96-Well Thermal Cycler (Applied Biosystem), using the following PCR profile; $95^{\circ} \mathrm{C}$ for $5 \mathrm{~min}, 35$ cycles of 1 $\min$ at $95^{\circ} \mathrm{C}, 1 \mathrm{~min}$ at $53^{\circ} \mathrm{C}$ and $1.30 \mathrm{~min}$ at $72^{\circ} \mathrm{C}$, and a final extension step of $8 \mathrm{~min}$ at $72^{\circ} \mathrm{C}$. The amplicons were analyzed by running on $1.8 \%$ agarose gel with $1 \mathrm{X}$ TAE at $80 \mathrm{~V}$ for $2 \mathrm{~h}$ using Bio-Rad gel electrophoresis unit. Amplified products were visualized using ethidium bromide under UVtransilluminator. The polymorphism information content (PIC), major allele frequency, heterozygosity and gene diversity were calculated for each marker using Power Marker ver. 3.25 (Liu and Muse, 2005). Principal co-ordinate analysis (PCoA) followed by cluster analysis was carried out using DARwin 6.0.14 (Perrier and Jacquemoud-Collet, 2006).

\section{Results and Discussion}

The ability of a marker to distinguish the genotypes (polymorphism information content, PIC) is an important indicator of polymorphism (Hildebrand et al., 1994), which is useful in genomic studies. The AhTE markers varied greatly for their PIC (Table 1), with an average PIC of 0.317 . Most of the markers recorded the PIC of 0.299 to 0.400 . But AhTE0251 and AhTE0487 recorded more than 0.400 PIC.The type(s) and frequency of alleles provide an idea about the major and minor alleles, the extent of heterozygous loci and population structure. The AhTE markers, by their virtue, are able to detect only two types of alleles (with and without AhMITE1) at a locus. In this study, the alleles without AhMITE1 insertion (B type) (0.56) were more frequent than those (A type) (0.44) with AhMITE1.The proportion of the major allele across the 79 AhTE marker loci ranged from 0.500 to 0.762 , with an average of 0.595 , indicating the overall abundance of the A type allele over the B type allele (Table 2).

For marker-trait association studies, the frequency of minor allele referring to the frequency at which the second most common allele occurs in a given population is important (Park et al., 2011) because the power of the statistical method encounters a challenge while discovering the lowfrequency $(\leq 0.05)$ phenotypic classes. Generally, an allele with a frequency of $5 \%$ or greater are targeted for genome-wide association studies. The minor allele frequency ranged from 0.238 to 0.504 with an average of 0.405 across the 79 AhTE marker loci (Table 2). Thus, the minor allele frequency in these populations allow the use of AhTE markers for genome-wide association studies. 
The observed heterozygosity $\left(H_{O}\right)$ for a locus denotes the proportion of the individuals heterozygous at that locus. It is related to the polymorphic nature of each locus. A high level of $\mathrm{Ho}$ at a locus generally correlates with high levels of genetic variation due to the adaptive response to environmental changes (Kotzé and Muller 1994). Our results showed a range of 0.000 to 0.240 with an average of 0.017 for $H_{O}$, indicating that on an average only $1.7 \%$ of the genotypes were heterozygous at each locus. No heterozygotes were observed at thirty-eight AhTE marker loci. Groundnut being a self-pollinated crop, a low level of observed heterozygosity is expected. However, certain Arachis species (e.g. A. glandulifera, A. helodes and $A$. kuhlmannii) showed moderate levels of observed heterozygosity (Bravo et al., 2006). A. helodes showed a heterozygosity of 0.0997 , indicating that, on average, $9.97 \%$ of individual plants were heterozygous for a locus. Further, the high $H_{O}$ in some species such as A. kuhlmannii (11.79\%) shows that allogamy or other mechanisms to retain heterozygosity occur in Arachis.

The allele frequency expected under HardyWeinberg equilibrium (HWE) is known as expected heterozygosity $\left(H_{E}\right)$. If the observed heterozygosity is lower than expected, the discrepancy can be attributed to forces such as inbreeding. If heterozygosity is higher than expected, an isolate-breaking effect (the mixing of two previously isolated populations) can be expected. As expected, $H_{O}(0.017)$ was much less than the $H_{E}(0.479)$. Such a high $H_{E}$ over $H_{O}$ were observed in the previous studies in groundnut (Palmieri et al., 2002) and chickpea (Sethy et al., 2006).

An attempt was made to compare the RIL and mutant population for the allele parameters. A difference in the minor allele frequency was observed between the RIL (0.488) and mutant (0.302) populations (Table 2 ). In addition, $H_{O}$ was more in the case of mutant population
(0.027) than in the RIL population (0.014). Because the genomic location of the AhTE markers was determined earlier, an effort was made to assess the population genetic parameters for the AhTE markers located on A genome with those present on $\mathrm{B}$ genome. The AhTE markers included 30 A genomespecific and 49B genome-specific markers. No major difference was observed between the $\mathrm{A}$ and $\mathrm{B}$ genome for the activity of AhMITE1. However, the B genome showed more activity of AhMITEl with mutagenesis over hybridization. The genic and intergenic location of the AhTE markers was already known, therefore an effort was made to compare the $H_{O}$ for the genic AhTE markers with the intergenic AhTE markers. The AhTE markers included 45 genic and 34 intergenic markers. $H_{O}$ was only $0.9 \%$ for the genic AhTE markers, while it was $2.7 \%$ for the intergenic markers, indicating a three-fold higher activity of AhMITEl in the intergenic region. Again, the mutant population showed higher $H_{O}$ than the RIL population, and it was more pronounced for intergenic (0.048) AhTE markers.

Using these genetic parameters, the principal co-ordinate analysis (PCoA) was attempted to estimate and visualize the similarity between the genotypes, where the genotypes ordinated closer to each other were more similar than those ordinated farther away. In this study, the average distance (dissimilarity) between the genotypes was 0.6866. Twenty pairs of genotypes showed the lowest dissimilarity of 0.0002 , while 120 pairs of the genotypes exhibited the highest dissimilarity (0.8598). The distance between the genotypes on graphical representation of the principal coordinate analysis confirmed the dissimilarity values (Figure 1). The genotypes showing the lowest dissimilarity failed to show any marker polymorphism, while those with the highest dissimilarity showed high polymorphism (up to $65.82 \%$ ). 
Table.1 Population genetic parameters for the 79 AhTE markers among the RIL and mutant populations of groundnut

\begin{tabular}{|c|c|c|c|c|c|c|}
\hline SN & Marker & \multicolumn{2}{|c|}{ Allele frequency } & $\boldsymbol{H}_{O}$ & $\boldsymbol{H}_{E}$ & PIC \\
\hline & & Major & Minor & & & \\
\hline 1 & AhTE0001 & 0.608 & 0.392 & 0.002 & 0.481 & 0.371 \\
\hline 2 & AhTE0005 & 0.736 & 0.264 & 0.000 & 0.389 & 0.313 \\
\hline 3 & AhTE0006 & 0.519 & 0.481 & 0.000 & 0.501 & 0.377 \\
\hline 4 & AhTE0010 & 0.707 & 0.293 & 0.126 & 0.416 & 0.331 \\
\hline 5 & AhTE0025 & 0.618 & 0.382 & 0.009 & 0.492 & 0.396 \\
\hline 6 & AhTE0032 & 0.506 & 0.494 & 0.000 & 0.507 & 0.386 \\
\hline 7 & AhTE0045 & 0.570 & 0.430 & 0.000 & 0.492 & 0.373 \\
\hline 8 & AhTE0050 & 0.534 & 0.466 & 0.023 & 0.508 & 0.390 \\
\hline 9 & AhTE0074 & 0.585 & 0.415 & 0.000 & 0.492 & 0.378 \\
\hline 10 & AhTE0101 & 0.513 & 0.487 & 0.000 & 0.503 & 0.380 \\
\hline 11 & AhTE0107 & 0.649 & 0.351 & 0.023 & 0.459 & 0.359 \\
\hline 12 & AhTE0113 & 0.548 & 0.452 & 0.017 & 0.505 & 0.389 \\
\hline 13 & AhTE0119 & 0.607 & 0.393 & 0.009 & 0.489 & 0.383 \\
\hline 14 & AhTE0121 & 0.497 & 0.503 & 0.028 & 0.511 & 0.392 \\
\hline 15 & AhTE0129 & 0.502 & 0.498 & 0.000 & 0.506 & 0.383 \\
\hline 16 & AhTE0130 & 0.638 & 0.362 & 0.015 & 0.479 & 0.385 \\
\hline 17 & AhTE0143 & 0.572 & 0.428 & 0.000 & 0.491 & 0.372 \\
\hline 18 & AhTE0148 & 0.555 & 0.445 & 0.011 & 0.505 & 0.390 \\
\hline 19 & AhTE0163 & 0.633 & 0.367 & 0.104 & 0.465 & 0.357 \\
\hline 20 & AhTE0164 & 0.598 & 0.402 & 0.019 & 0.482 & 0.368 \\
\hline 21 & AhTE0189 & 0.536 & 0.464 & 0.000 & 0.497 & 0.374 \\
\hline 22 & AhTE0191 & 0.529 & 0.471 & 0.002 & 0.504 & 0.382 \\
\hline 23 & AhTE0212 & 0.560 & 0.440 & 0.000 & 0.494 & 0.374 \\
\hline 24 & AhTE0218 & 0.519 & 0.481 & 0.011 & 0.499 & 0.375 \\
\hline 25 & AhTE0222 & 0.508 & 0.492 & 0.057 & 0.507 & 0.386 \\
\hline 26 & AhTE0232 & 0.694 & 0.306 & 0.000 & 0.424 & 0.334 \\
\hline 27 & AhTE0233 & 0.502 & 0.498 & 0.000 & 0.500 & 0.375 \\
\hline 28 & AhTE0237 & 0.540 & 0.460 & 0.000 & 0.499 & 0.376 \\
\hline 29 & AhTE0245 & 0.594 & 0.406 & 0.019 & 0.484 & 0.369 \\
\hline 30 & AhTE0249 & 0.517 & 0.483 & 0.000 & 0.501 & 0.377 \\
\hline 31 & AhTE0251 & 0.543 & 0.457 & 0.000 & 0.529 & 0.425 \\
\hline 32 & AhTE0254 & 0.598 & 0.402 & 0.019 & 0.481 & 0.365 \\
\hline 33 & AhTE0261 & 0.558 & 0.442 & 0.000 & 0.496 & 0.377 \\
\hline 34 & AhTE0278 & 0.665 & 0.335 & 0.006 & 0.449 & 0.353 \\
\hline 35 & AhTE0283 & 0.592 & 0.408 & 0.000 & 0.487 & 0.374 \\
\hline 36 & AhTE0296 & 0.592 & 0.408 & 0.023 & 0.487 & 0.374 \\
\hline 37 & AhTE0303 & 0.629 & 0.371 & 0.025 & 0.471 & 0.365 \\
\hline 38 & AhTE0305 & 0.562 & 0.438 & 0.000 & 0.500 & 0.384 \\
\hline
\end{tabular}


Int.J.Curr.Microbiol.App.Sci (2017) 6(8): 2051-2060

\begin{tabular}{|c|c|c|c|c|c|c|}
\hline 39 & AhTE0317 & 0.719 & 0.281 & 0.008 & 0.404 & 0.323 \\
\hline 40 & AhTE0324 & 0.575 & 0.425 & 0.004 & 0.507 & 0.399 \\
\hline 41 & AhTE0328 & 0.526 & 0.474 & 0.125 & 0.502 & 0.380 \\
\hline 42 & AhTE0332 & 0.668 & 0.332 & 0.000 & 0.445 & 0.348 \\
\hline 43 & AhTE0335 & 0.588 & 0.412 & 0.017 & 0.498 & 0.390 \\
\hline 44 & AhTE0357 & 0.570 & 0.430 & 0.000 & 0.493 & 0.375 \\
\hline 45 & AhTE0359 & 0.599 & 0.401 & 0.009 & 0.491 & 0.383 \\
\hline 46 & AhTE0369 & 0.658 & 0.342 & 0.011 & 0.457 & 0.363 \\
\hline 47 & AhTE0373 & 0.682 & 0.318 & 0.021 & 0.438 & 0.349 \\
\hline 48 & AhTE0381 & 0.632 & 0.368 & 0.000 & 0.465 & 0.357 \\
\hline 49 & AhTE0391 & 0.594 & 0.406 & 0.000 & 0.485 & 0.371 \\
\hline 50 & AhTE0416 & 0.557 & 0.443 & 0.064 & 0.499 & 0.380 \\
\hline 51 & AhTE0433 & 0.688 & 0.312 & 0.002 & 0.434 & 0.346 \\
\hline 52 & AhTE0437 & 0.658 & 0.342 & 0.019 & 0.452 & 0.353 \\
\hline 53 & AhTE0465 & 0.666 & 0.334 & 0.000 & 0.445 & 0.346 \\
\hline 54 & AhTE0470 & 0.715 & 0.285 & 0.004 & 0.409 & 0.327 \\
\hline 55 & AhTE0477 & 0.670 & 0.330 & 0.000 & 0.446 & 0.351 \\
\hline 56 & AhTE0482 & 0.509 & 0.491 & 0.000 & 0.509 & 0.389 \\
\hline 57 & AhTE0483 & 0.634 & 0.366 & 0.000 & 0.468 & 0.364 \\
\hline 58 & AhTE0486 & 0.608 & 0.392 & 0.000 & 0.481 & 0.371 \\
\hline 59 & AhTE0487 & 0.581 & 0.419 & 0.000 & 0.506 & 0.401 \\
\hline 60 & AhTE0491 & 0.575 & 0.425 & 0.026 & 0.489 & 0.369 \\
\hline 61 & AhTE0498 & 0.500 & 0.500 & 0.000 & 0.504 & 0.381 \\
\hline 62 & AhTE0501 & 0.528 & 0.472 & 0.000 & 0.504 & 0.382 \\
\hline 63 & AhTE0517 & 0.579 & 0.421 & 0.004 & 0.489 & 0.371 \\
\hline 64 & AhTE0523 & 0.762 & 0.238 & 0.008 & 0.363 & 0.299 \\
\hline 65 & AhTE0524 & 0.519 & 0.481 & 0.000 & 0.503 & 0.380 \\
\hline 66 & AhTE0536 & 0.625 & 0.375 & 0.000 & 0.477 & 0.374 \\
\hline 67 & AhTE0540 & 0.677 & 0.323 & 0.004 & 0.437 & 0.342 \\
\hline 68 & AhTE0550 & 0.643 & 0.357 & 0.174 & 0.460 & 0.356 \\
\hline 69 & AhTE0552 & 0.609 & 0.391 & 0.015 & 0.482 & 0.373 \\
\hline 70 & AhTE0563 & 0.664 & 0.336 & 0.000 & 0.447 & 0.349 \\
\hline 71 & AhTE0571 & 0.592 & 0.408 & 0.013 & 0.497 & 0.389 \\
\hline 72 & AhTE0572 & 0.685 & 0.315 & 0.000 & 0.433 & 0.341 \\
\hline 73 & AhTE0586 & 0.553 & 0.447 & 0.000 & 0.501 & 0.383 \\
\hline 74 & AhTE0590 & 0.553 & 0.447 & 0.000 & 0.503 & 0.385 \\
\hline 75 & AhTE0599 & 0.596 & 0.404 & 0.000 & 0.496 & 0.390 \\
\hline 76 & AhTE0607 & 0.599 & 0.401 & 0.002 & 0.483 & 0.370 \\
\hline 77 & AhTE0630 & 0.646 & 0.354 & 0.240 & 0.459 & 0.355 \\
\hline 78 & AhTE0661 & 0.552 & 0.448 & 0.002 & 0.498 & 0.378 \\
\hline 79 & AhTE0674 & 0.500 & 0.500 & 0.000 & 0.507 & 0.386 \\
\hline
\end{tabular}


Table.2 Population genetic parameters for the AhTE markers among the RIL and mutant populations of groundnut

\begin{tabular}{|c|c|c|c|c|c|c|c|c|c|c|}
\hline Population & \multicolumn{2}{|c|}{$\begin{array}{c}\text { Major allele } \\
\text { frequency }\end{array}$} & \multicolumn{2}{|c|}{$\begin{array}{c}\text { Minor } \\
\text { allele frequency }\end{array}$} & \multicolumn{2}{|c|}{ Gene diversity } & \multicolumn{2}{|c|}{ Heterozygosity } & \multicolumn{2}{|c|}{$\begin{array}{c}\text { Polymorphism } \\
\text { information content }\end{array}$} \\
\hline \multicolumn{11}{|c|}{ All AhTE markers (79) } \\
\hline Mutant population & 0.698 & $0.510-0.989$ & 0.302 & $0.010-0.490$ & 0.390 & $0.020-0.500$ & 0.027 & $0.000-0.674$ & 0.308 & $0.020-0.375$ \\
\hline Pooled population & 0.595 & $0.500-0.762$ & 0.405 & $0.238-0.500$ & 0.479 & 0.363-0.529 & 0.017 & $0.000-0.240$ & 0.317 & $0.299-0.425$ \\
\hline Mutant population & 0.740 & $0.510-0.990$ & 0.260 & $0.010-0.490$ & 0.352 & $0.020-0.500$ & 0.012 & $0.000-0.153$ & 0.282 & $0.020-0.375$ \\
\hline Pooled population & 0.621 & $0.500-0.736$ & 0.379 & $0.264-0.500$ & 0.469 & $0.389-0.508$ & 0.019 & $0.000-0.240$ & 0.365 & 0.313-0.399 \\
\hline \multicolumn{11}{|c|}{ B genome AhTE markers (49) } \\
\hline Mapping population & 0.569 & $0.501-0.730$ & 0.431 & $0.270-0.499$ & 0.491 & $0.395-0.536$ & 0.010 & $0.000-0.155$ & 0.378 & $0.319-0.435$ \\
\hline Mutant population & 0.672 & 0.510-0.929 & 0.328 & $0.071-0.490$ & 0.414 & $0.133-0.500$ & 0.036 & $0.000-0.674$ & 0.324 & $0.124-0.375$ \\
\hline Pooled population & 0.605 & $0.502-0.762$ & 0.395 & $0.238-0.498$ & 0.474 & $0.363-0.529$ & 0.009 & $0.000-0.174$ & 0.368 & $0.299-0.425$ \\
\hline \multicolumn{11}{|c|}{ Intergenic AhTE markers (34) } \\
\hline Mapping population & 0.570 & $0.502-0.663$ & 0.498 & $0.337-0.498$ & 0.492 & $0.448-0.515$ & 0.022 & $0.000-0.259$ & 0.378 & $0.350-0.408$ \\
\hline Mutant population & 0.677 & $0.520-0.939$ & 0.323 & $0.061-0.480$ & 0.414 & $0.115-0.499$ & 0.048 & $0.000-0.674$ & 0.324 & $0.108-0.375$ \\
\hline Pooled population & 0.581 & $0.500-0.707$ & 0.419 & $0.293-0.500$ & 0.485 & $0.416-0.511$ & 0.027 & $0.000-0.240$ & 0.373 & $0.331-0.399$ \\
\hline
\end{tabular}


Fig.1 Principal co-ordinates (a) and clusters for the genotypes (b)
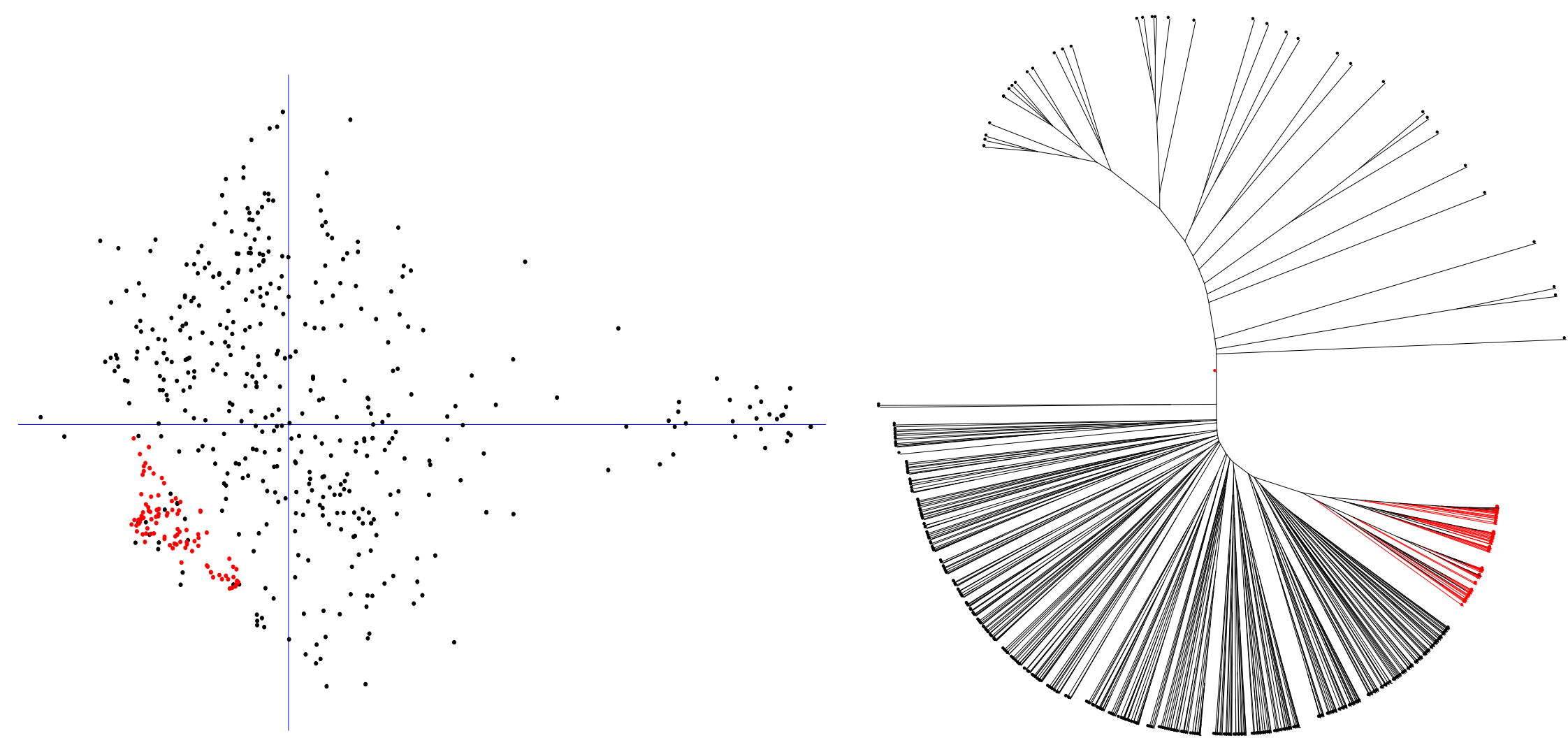

RILs of TMV $2 \times$ TMV 2-NLM

populationMutant population 
The genotypes were also subjected to cluster analysis using DARwin. Two diverse groups could be clearly distinguished (Figure 2). One small group consisted of only RILs, and the other large group contained both RILs and the genotypes of mutant population.

To conclude, the AhTE markers which detected the polymorphism due to AhMITE1 insertion both in genic and intergenic regions of groundnut genome, showed high PIC and polymorphism. AhTE markers could be successfully used to group the genotypes based on the principal co-ordinates. Therefore, AhTE markers might provide an efficient marker system, like SSR markers (Li et al., 2011; Ren et al., 2014), for genetic and genomic studies in groundnut. Recently, AhTE markers have been successfully employed for trait mapping in groundnut (Shirasawa et al., 2012b; Kolekar et al., 2016). They were also used to identify and clone the gene governing various traits (Lee et al., 2006; Monden et al., 2009; Sato et al., 2013) since the transposable element served as a DNA tag.

\section{References}

Bertioli, D.J., S.B. Cannon, L. Froenicke, G. Huang, A.D. Farmer, E.K. Cannon, X. Liu, D. Gao, J. Clevenger, S. Dash, L. Ren, M.C. Moretzsohn, K. Shirasawa, W. Huang, B. Vidigal, B. Abernathy, Y. Chu, C.E. Niederhuth, P. Umale, A.C. Araujo, A. Kozik, K. Do Kim, M.D. Burow, R.K. Varshney, X. Wang, X. Zhang, N. Barkley, P.M. Guimaraes, S. Isobe, B. Guo, B. Liao, H.T. Stalker, R.J. Schmitz, B.E. Scheffler, S.C. LealBertioli, X. Xun, S.A. Jackson, R. Michelmore and P. Ozias-Akins, 2016, The genome sequences of Arachis duranensis and Arachis ipaensis, the diploid ancestors of cultivated peanut. Nat. Genet., 48: 438-446.
Bevan, M.W., C. Uauy, B.B. Wulff, J. Zhou, K. Krasileva and M.D. Clark, 2017, Genomic innovation for crop improvement. Nature, 543: 346-354.

Bhat, R.S., V.U. Patil, T.M. Chandrashekar, V. Sujay, M.V.C. Gowda and M.S. Kuruvinashetti, 2008, Recovering flanking sequence tags of miniature inverted-repeat transposable element by thermal asymmetric interlaced-PCR in peanut. Curr. Sci., 95: 452-453.

Bravo, J.P., A.A. Hoshino, C.M. Angelici, C.R. Lopes and M.A. Gimenes, 2006, Transferability and use of microsatellite markers for the genetic analysis of the germplasm of some Arachis section species of the genus Arachis. Genet. Mol. Biol., 29: 516-524.

Chen, X., H. Li, M.K. Pandey, Q. Yang, X. Wang, V. Garg, X. Chi, D. Doddamani, Y. Hong, H. Upadhyaya, H. Guo, A.W. Khan, F. Zhu, X. Zhang, L. Pan, G.J. Pierce, G. Zhou, K.A. Krishnamohan, M. Chen, N. Zhong, G. Agarwal, S. Li, A. Chitikineni, G.Q. Zhang, S. Sharma, N. Chen, H. Liu, P. Janila, M. Wang, T. Wang, J. Sun, X. Li, C. Li, L. Yu, S. Wen, S. Singh, Z. Yang, J. Zhao, C. Zhang, Y. Yu, J. Bi, Z.J. Liu, A.H. Paterson, S. Wang, X. Liang, R.K. Varshney and S. Yu, 2016, Draft genome of the peanut A-genome progenitor (Arachis duranensis) provides insights into geocarpy, oil biosynthesis, and allergens. Proc. Natl. Acad. Sci., 113: 6785-6790.

Gowda, M.V.C., R.S. Bhat, B.N. Motagi, V. Sujay, Varshakumari and S. Bhat, 2010, Association of high-frequency origin of late leaf spot resistant mutants with AhMITE1 transposition in peanut. Plant Breed, 129: 567-569.

Hildebrand, C.E., C. David, C. Torney and P. Wagner (eds) 1994. Informativeness of polymorphic DNA markers, Vol. University Science Books, Mill Valley, 
California

Holbrook, C.C., P. Ozias-Akins, Y. Chu and B. Guo, 2011, Impact of molecular genetic research on peanut cultivar development. Agron., 1: 3-17.

Khera, P., H.D. Upadhyaya, M.K. Pandey, M. Roorkiwal, M. Sriswathi, P. Janila, Y. Guo, M.R. McKain, E.D. Nagy and S.J. Knapp, 2013, Single nucleotide polymorphism-based genetic diversity in the reference set of peanut (spp.) by developing and applying cost-effective Kompetitive Allele Specific Polymerase Chain Reaction genotyping assays. Plant Genome, 6.

Kolekar, R.M., V. Sujay, K. Shirasawa, M. Sukruth, Y.P. Khedikar, M.V.C. Gowda, M.K. Pandey, R.K. Varshney and R.S. Bhat, 2016, QTL mapping for late leaf spot and rust resistance using an improved genetic map and extensive phenotypic data on a recombinant inbred line population in peanut (Arachis hypogaea L.). Euphytica, 209: 147-156.

Kotzé, A. and G.H. Muller. 1994. Genetic relationship in South African cattle breeds, Proceedings of the 5th world congress on genetics applied to livestock production, University of Guelph, Guelph, Canada, p 413-416.

Lee, J.K., J.Y., Park, J.H. Kim, S.J. Kwon, J.H. Shin, S.K. Hong, H.K. Min and N.S. Kim, 2006, Genetic mapping of the Isaac-CACTA transposon in maize. Theor. Appl. Genet., 113: 16-22.

Li, Y., C. Chen, S. Knapp, A. Culbreath, C. Holbrook and B. Guo, 2011, Characterization of simple sequence repeat (SSR) markers and genetic relationships within cultivated peanut (Arachis hypogaea L.). Peanut Sci., 38: $1-10$.

Liu, K. and S.V. Muse. 2005. PowerMarker: an integrated analysis environment for genetic marker analysis. Bioinformatics,
21: 2128-2129.

Mace, E.S., K.K. Buhariwalla, H.K. Buhariwalla and J.H. Crouch, 2003, A high-throughput DNA extraction protocol for tropical molecular breeding programs. Plant Mol. Biol. Rep., 21: 459-460.

Monden, Y., K. Naito, Y. Okumoto, H. Saito, N. Oki, T. Tsukiyama, O. Ideta, T. Nakazaki, S.R. Wessler and T. Tanisaka, 2009, High potential of a transposon mPing as a marker system in japonica $\times$ japonica cross in rice. DNA Res., 16: 131-140.

Moretzsohn, M.C., A.V.G. Barbosa, D.M.T. Alves-Freitas, C. Teixeira, S. LealBertioli, P.M. Guimaraes, R.W. Pereira, C.R. Lopes, M.M. Cavallari and J.F.M. Valls, 2009, A linkage map for the Bgenome of Arachis (Fabaceae) and its synteny to the A-genome. BMC Plant Biol., 9: 40.

Palmieri, D.A., A. Hoshino, J. Bravo, C. Lopes and M. Gimenes, 2002, Isolation and characterization of microsatellite loci from the forage species Arachis pintoi (Genus Arachis). Mol. Ecol. Notes, 2: 551-553.

Park, J.H., M.H. Gail, C.R. Weinberg, R.J. Carroll, C.C. Chung, Z. Wang, S.J. Chanock, J.F. Fraumeni and N. Chatterjee, 2011, Distribution of allele frequencies and effect sizes and their interrelationships for common genetic susceptibility variants. Proc. Natl. Acad. Sci., 108: 18026-18031.

Perrier, X. and J. Jacquemoud-Collet, P. 2006. DARwin software. http//darwinciradfr/darwin:

Ren, X., H. Jiang, Z. Yan, Y. Chen, X. Zhou, L. Huang, Y. Lei, J. Huang, L. Yan and Y. Qi, 2014, Genetic diversity and population structure of the major peanut (Arachis hypogaea L.) cultivars grown in China by SSR markers. PLoS One, 9: e88091. 
Sato, K., Y. Mukainari, K. Naito and K. Fukunaga, 2013, Construction of a foxtail millet linkage map and mapping of spikelet-tipped bristles1 (stbl) by using transposon display markers and simple sequence repeat markers with genome sequence information. Mol. Breed 31: 675-684.

Sethy, N.K., B. Shokeen, K.J. Edwards and S. Bhatia. 2006. Development of microsatellite markers and analysis of intraspecific genetic variability in chickpea (Cicer arietinum L.). Theor. Appl. Genet., 112: 1416-1428.

Shirasawa, K., H. Hirakawa, S. Tabata, M. Hasegawa, H. Kiyoshima, S. Suzuki, S. Sasamoto, A. Watanabe, T. Fujishiro and S. Isobe, 2012a, Characterization of active miniature inverted-repeat transposable elements in the peanut genome. Theor. Appl. Genet., 124: 1429-1438.

Shirasawa, K., P. Koilkonda, K. Aoki, H. Hirakawa, S. Tabata, M. Watanabe, M.
Hasegawa, H. Kiyoshima, S. Suzuki, C. Kuwata, Y. Naito, T. Kuboyama, A. Nakaya, S. Sasamoto, A. Watanabe, M. Kato, K. Kawashima, Y. Kishida, M. Kohara, A. Kurabayashi, C. Takahashi, H. Tsuruoka, T. Wada and S. Isobe, $2012 \mathrm{~b}$, In silico polymorphism analysis for the development of simple sequence repeat and transposon markers and construction of linkage map in cultivated peanut. BMC Plant Biol., 12: 80.

Song, G.Q., M.J. Li, H. Xiao, X.J. Wang, R.H. Tang, H. Xia, C.Z. Zhao and Y.P. $\mathrm{Bi}, 2010$, EST sequencing and SSR marker development from cultivated peanut (Arachis hypogaea L.). Electron J. Biotechnol., 13: 7-8.

Yuan, Y., P.E. Bayer, J. Batley and D. Edwards. 2017. Improvements in genomic technologies: application to crop genomics. Trends Biotechnol., 35: 547-558.

\section{How to cite this article:}

Hake, A.A. and Bhat, R.S. 2017. Utility of AhTE Markers for Genetic and Genomic Studies in Groundnut (Arachis hypogaea L.). Int.J.Curr.Microbiol.App.Sci. 6(8): 2051-2060. doi: https://doi.org/10.20546/ijcmas.2017.608.244 